\title{
Preparing for the future: development of an 'antifragile' methodology that complements scenario planning by omitting causation.
}

\begin{abstract}
This paper demonstrates that the Intuitive Logics method of scenario planning emphasises the causal unfolding of future events and that this emphasis limits its ability to aid preparation for the future, for example by giving a misleading impression as to the usefulness of 'weak signals' or 'early warnings'. We argue for the benefits of an alternative method that views uncertainty as originating from indeterminism. We develop and illustrate an 'antifragile' approach to preparing for the future and present it as a step-by-step, non-deterministic methodology that can be used as a replacement for, or as a complement to, the causally-focused approach of scenario planning.
\end{abstract}

Keywords: scenario planning; Intuitive Logics; causality; indeterminism; fragility; uncertainty

\section{Introduction}

Scenario planning exercises are increasingly common in the private sector and are increasingly underpinned by academic research [1, 2 p.461, 3, 4 p.335]. While this increased popularity has been accompanied by a proliferation of approaches [5], it is widely accepted that most organizations employing scenario planning use an approach based on what is known as 'Intuitive Logics' (IL) [1, 6 p.9, 7 p.162]. Here, management team members are facilitated in thinking of the causal unfolding of chains of events into the future. 
However, questions have been raised about IL's effectiveness in preparing an organization for the future. In particular, much has recently been written highlighting its limited ability to deal with uncertainty - particularly its ability to assist in preparing for 'surprise' events [8-10].

In discussing this limitation, we present two interpretations of the origins of uncertainty. One view associates uncertainty with a difficulty in identifying and predicting causes and their effects but, nevertheless, still sees events as determined by causes. This is contrasted with an alternative view of uncertainty that rejects cause altogether and views events as subject to an absolute form of indeterminism. The distinction between these two interpretations is important because it allows evaluation of the appropriateness and efficacy of the methods currently used to carry out scenario planning.

Our paper demonstrates that the basic IL methodology is based closely on the deterministic interpretation of uncertainty but that a recent augmentation, using 'backwards logic', has, to a degree, broadened this base assumption. We argue that, in order to provide a full recognition of uncertainty, methods and approaches that are non-deterministic must now be developed. In other words, scenario planning must, in our view, weaken its dependence on causation. In the latter sections of the paper we develop and present a new approach for preparing for the future without recourse to causality. First, we develop our arguments on conceptualisations of uncertainty.

\section{Scenario planning and the nature of uncertainty}


There are two major interpretations of the origins of uncertainty. One interpretation views it as the result of 'ontological randomness' and this is associated with the emergence of quantum mechanics in the early $20^{\text {th }}$ century. Here, observable phenomena are seen as subject to an irreducible and absolute form of chance - as in Brownian motion in physics [11]. Under this view, prediction is impossible. Such indeterminism is a rejection of the very notion of cause, the only 'cause' being chance itself. A particular combination of factors may result in one outcome on one occasion and a completely different outcome on another. Hacking, a philosopher of science, commented regarding the advent of this view that 'Causality, long the bastion of metaphysics, was toppled, or at least tilted' [12].

An alternative interpretation of the origins of uncertainty views it as the result of 'epistemological randomness'. This viewpoint originated in 1814 from Laplace's scientific determinism [13, 14, 15 p.37]. Under this view, uncertainty stems from the difficulty associated with identifying, unravelling and measuring causes, but events are nevertheless still seen as determined by cause. Uncertainty results from the inadequacy of the procedures we use to uncover cause, which can have limited efficacy, even ex-post. Nevertheless, this deterministic view suggests that if we are able to identify relevant causes at a sufficiently early stage in their development we can alter the expected outcome by changing the causes. Under this view, each future outcome results from a specific set of causes and is determined by them.

Taleb [16 p.198] refers to these alternative interpretations as 'true randomness' and 'deterministic chaos', respectively. He implies that there is no functional difference between them if it is, in practice, impossible to locate relevant causes. If we believe 
that events are entirely determined by causes but that these causes cannot be identified in advance (or, indeed, even retrospectively), then uncertainty should, by default, be considered to be ontological in origin.

This distinction between the two interpretations of the origins of uncertainty, one which emphasises determinism and one which rejects it, is important because it enables a nuanced evaluation of the methods used for scenario planning. As shown by Shackle [17], deterministic approaches to the future are of limited value in contexts characterised by novelty and surprise. In fact, a limited ability to deal with 'surprise' events has been identified as a deficiency in the currently most-commonly employed method of scenario planning, IL [8-10].

To overcome this limitation, approaches to scenario planning should, we believe, accept indeterminism as a complement to an acceptance of 'cause'. As commented on by Loasby [18 p.2-4] in relation to the hole in the ozone layer discovered in the 1980s, any human notion of cause can only ever be provisional and tentative in nature. The fluorine compounds that caused the damage to the ozone were earlier thought to be inert. It only later transpired that this inertness, established in the laboratory setting, did not apply in the ozone layer. This limited capacity to generalise cause from studies in the experimental settings of the natural sciences is indicative of much larger difficulties in ascribing cause in the social realm.

It is not always possible to know the extent to which experienced reality is genuinely subject to indeterminism stemming from 'true randomness' compared to the extent to which it simply appears so because of our limited ability to identify cause. Phillips 
[51 p.5] provides an example illustrating this difficulty: Biologists studying predatorprey relationships thought that the extinction of a species must always have a 'cause' and could not understand how it could happen to a predator when its prey was plentiful. They later realised, however, that extinction can happen for no apparent reason.

By adopting an ontological interpretation of uncertainty - and assuming indeterminism - we can augment scenario methodology to take account of this indeterminism. By contrast, the cause-and-effect logic of IL results in the making of multiple causal assumptions and so contains multiple opportunities for error and does not take account of indeterminism.

The later sections of this paper outline Taleb's alternative 'Antifragile' (AF) [19] approach to aid preparation for the future. We will show that use of the AF conceptualisation can enhance preparation for a future of deep uncertainty without making recourse to causal assumptions. We develop and document AF techniques and methods as a complement to, or as an alternative to, a formalised scenarioplanning procedure.

Prior to this, however, we (i) substantiate our claim as to the deterministic nature of IL, and (ii) evidence the negative effect of this determinism on IL's ability to deal with uncertainty. The next section describes IL's inherent determinism and some of its negative effects.

\section{Determinism in scenario planning and its effects}


3.1 The determinism of the Intuitive Logics scenario methodology

Postma and Liebl [7] have provided a basic outline of the IL approach to scenario planning. In the first part of the IL process, the 'driving forces' or 'causal factors' assumed to determine (degree of) change in the future are classified into the three categories of 'constant', 'predetermined' or 'uncertain'. The 'constant' group of factors are characterised by their lack of change and represent a continuation of the present. For 'predetermined' factors of change, change is assumed to occur but the change is known and predictable. The third category of factors is for those which are 'uncertain'. It is this third category that is most important as it is from these uncertainties that a range of scenarios are produced in the IL process.

The driving forces are clustered based on degree of interdependence and the two clusters considered to have both (i) the highest uncertainty and (ii) the highest degree of potential impact on the focal issue of concern to the scenario planners are utilised as the basis of the two-dimensional scenario space (Fig. 1) from which four scenarios are usually created, one for each quadrant comprising the space [8]. These four scenarios are developed from the cluster contents in the form of 'pen-pictures' $[10$ p.363] - narratives in which a chain of causation is described resulting in an 'end state', or ultimate outcome. A final step of the process examines the robustness of the organization's strategy against the developed scenarios [9 p.817].

Indeterminism is incorporated into this process through the acknowledgement of multiple possible futures. However, each one of these futures is individually tied to a specific set of causes and effects and is entirely determined by these causes and effects. Each scenario is deterministic in its own right, even if the overall process is 
non-deterministic since four scenarios emerge rather than one. A specific unfolding future is a result of an interdependent set of causes, a 'causal chain'.

The IL scenario generation process generates rich, qualitative, and engaging stories of these individually-determined futures - based on a causal logic. The resulting narrative represents a 'sequence of interacting events needed to reach the scenario' outcome [21 p.224, 22]. As vant' Klooster and van Asselt [23 p.23] specifically state, the four scenario 'stories' resulting from an IL scenario-planning exercise are, at their heart, 'based on a cause and effect logic'. It is this logic, and its associated determinism, that places IL within the categorisation of a deterministic, epistemological interpretation of the origins of uncertainty.

\section{INSERT FIGURE 1 ABOUT HERE}

\subsection{Deleterious effects of a deterministic focus in scenario planning}

IL's grounding in the deterministic, epistemological view of uncertainty manifests itself in a dependence on narratives describing chains of cause-and-effect logic. This focus has a number of effects on its efficacy in relation to uncertainty, as will be shown in this section.

Taleb [16] has given the human tendency to over-emphasise the role of causal factors in any particular outcome the name of 'Narrative Fallacy' (NF). An example of NF provided by Taleb [16] is the way in which history is written. When living through or participating in an historical incident the course of unfolding events will seem very complex and messy. Yet, when reading back through the history of the same incident as later documented by the historian, the eventual outcome appears clearly determined 
by an identifiable set of more-or-less important causes [25 p.87-108]. Participants in IL engage in an analogous process when constructing scenarios.

Taleb argues that, in contrast to the neat categorisation and ranking of causes by the historian, outcomes are much less determined by causes than they appear in the history books. As Taleb [16 p.63-64] comments, narratives bind facts together making them more easily remembered and prominent in the minds of readers, as well as making them easier to understand.

IL uses narrative to enhance the plausibility of the sequence of events and actions within individual scenarios. This is a commonly asserted benefit of the narrativebased approach to scenario planning incorporated in IL. Storytelling via the causeand-effect sequences within the IL version of scenario planning may be a natural way of making sense of the world as evidenced by the similar use of narrative in the decision-making processes of judges and juries as shown by Wagenaar [24]. In this way, the IL methodology can, it is argued, act to 'minimise unpleasant surprises' [21 p.224] by using the natural pre-disposition to construct and think in narrative terms in order to raise the prominence and plausibility of futures not previously considered.

However, the IL scenario process can also have the opposite effect of increasing susceptibility to unpleasant surprises. The IL process enhances the plausibility of futures other than a 'best guess' future that is often assumed by decision-makers, but it only does so for those scenarios considered as part of the scenario-planning exercise. The corollary is that unconsidered futures, which are much larger in number, are likely to be diminished in terms of their plausibility. 
IL therefore has the effect of both broadening and narrowing decision-makers' perspective at the same time. However, since the IL process typically results in just four scenarios, whereas the number of unconsidered scenarios is infinite in number (and, therefore, much more likely to be the source of the actual future that transpires), on balance its perspective-narrowing effect may be considerably greater than its perspective-broadening effect. This narrowing will result directly from the employment of narratives describing chains of causation, which in turn stems from IL's grounding in a deterministic, epistemological view of uncertainty.

The very act of producing scenarios can give participants undue confidence in their ability to predict $[8-9,26]$ even when the scenario-planning process is explicitly couched in terms of a non-predictive exercise. Drawing on Tversky and Kahneman [27], Wright and Goodwin [9 p.818] refer to this effect as the 'simulation heuristic'. The simulation heuristic comes about in particular because of the so-called 'conjunction fallacy' which is directly implicated in the use of narratives to describe chains of causation and, therefore, to IL's grounding in the epistemological interpretation of uncertainty. As Kahneman [28 p.159-160] has shown, richer and more detailed descriptions of the causal intersections of several events are, psychologically, seen as more likely than the base probabilities of the constituent events. By the formal probability laws, the intersection of the occurrence of two independent events can only ever be lower than the probability of occurrence of either one individually. 
The simulation heuristic results in a greater perceived plausibility and likelihood of occurrence for considered scenarios. In this way, the perspective-narrowing effect that results from the very act of creating specific narratives that are based on a sub-section of identified driving forces is magnified. This can act to make organizations and their managers more rather than less susceptible to 'surprise' futures not considered as part of the focal scenarios.

Because of the emphasis on narrative and cause in the IL process, the impression can develop that the way to deal with uncertainty is simply to identify the unfolding of constructed causal chains at as early a stage as possible, for example by identifying early 'trigger events', or 'weak signals' [29] or 'early warnings' - and then to put in place contingencies in order to mitigate the unfolding of an undesirable future or take early advantage of the unfolding of a desirable future. This view is prominent in the recent scenario-planning literature [30-31].

An ontological view of uncertainty, by contrast, views outcomes as resulting from stochastic processes that are not connected with causation. This conceptualisation implies that scanning for 'weak signals' or 'early warnings' will lead to misplaced confidence in the unfolding of the particular focal futures described in the constructed scenarios.

3.3 A recent attempt to overcome determinism in scenario planning: the backwards logic methodology

One attempt to broaden the range of scenarios developed within the general IL methodology is Wright and Goodwin's [9] Backwards Logic Method (BLM). BLM is 
an adaptation and augmentation of IL designed to broaden out the range of scenarios considered 'while, at the same time, retaining the essential focus on causality within the process of scenario construction' [8 p.638, emphasis added]. Within the BLM, the focus is shifted onto understanding the causes of plausible changes to the organization's achievement of its objectives.

Essentially, BLM has three steps, with an iterative fourth step designed to consolidate the process and ensure that extreme events are considered. Step 1 identifies the organization's key objectives, step 2 imagines a range of extreme outcomes for those objectives, and step 3 establishes the factors that could cause these changes (identified in step 2) in the achievement of objectives. Crucially, step 3 retains the focus on building narratives that result in a causal chain, albeit one that runs backwards from the future achievement, or otherwise, of organizational objectives to the events causing that variation in achievement.

As evident in our description of step 3, BLM still imposes an arrow of causation but sees this arrow running backwards in time - identifying the reverse causal chain that results in the over-achievement or under-achievement of objectives. As described in section two, such an arrow of causation, or unfolding causal chain is, from Taleb's viewpoint, a likely 'narrative fallacy' whose very construction excludes indeterminism and enhances a view of the future as determined. This is true regardless of in which direction the arrow, or chain of causation, travels.

Because of the continued dependence on narratives describing chains of causation the considered scenarios will still gain in prominence at the expense of those left 
unconsidered. The effect may be an increase, rather than a decrease, in susceptibility to 'unpleasant surprises', though this effect is likely to be less prevalent with BLM than under IL because of the former's initial focus on extreme changes in the achievement of an organization's key objectives. In fact, the originators of the BLM approach were aware of the perspective-limiting bias in standard applications of IL when they proposed BLM and, for this reason included in it suggestions to bolster the process to ensure it is not one solely based on describing the unfolding of causal chains $[9, \mathrm{p} .821]$.

Nevertheless, the BLM approach retains a philosophical grounding that is closer to the deterministic, epistemological view of uncertainty than the ontological. It still implies that the way to prepare for 'extreme' events is through an identification of what might cause them. Because of its continued grounding in the deterministic, epistemological view, specific sets of causes are still assumed to result in a single outcome (under-achievement, achievement, or over-achievement of an objective).

\section{Preparing for the future without recourse to cause: Antifragility}

The previous sections of this paper have shown IL to be grounded in a deterministic, epistemological view of the origin of uncertainty. The negative consequences of this have been discussed and it has been shown that a recent augmentation of IL, BLM, attempts to mitigate these problems. We next turn to consider and evaluate alternative, non-scenario-based, approaches to aid preparation for the future.

Taleb's 'Antifragile' (AF) approach to preparation for an uncertain future [19] does not require causal constructions of what may, or may not, initiate a particular future 
outcome and so attenuates our potential fallibility in identification of cause, discussed earlier. In short, causal attributions cannot be misplaced if none are made.

In switching emphasis onto what makes the organization (or individual) 'fragile' or sensitive to the future, AF may initially be considered similar to BLM. For example, BLM like AF seeks to identify the factors that may result in a failure to meet objectives and contains techniques to avoid misattributing cause [9 p.823]. However, while BLM seeks to minimise the possibility of misattributing cause, AF seeks to omit cause altogether, and this is an important distinction.

A second important distinction is that AF shifts the conceptual focus onto both withstanding high impact, 'surprise' events and benefitting from their occurrence. This distinction delineates $\mathrm{AF}$ as something more than an approach to enable protection from the worst effects of 'negatively-valenced' events whilst enabling benefit from 'positively-valenced' events - suggested by Goodwin and Wright as perhaps the only way to deal with deep uncertainty [10 p.367].

We next present the main components of the AF approach, as developed by Taleb, and outline how these can be combined to constitute an approach that can either be implemented as a complement to scenario methodologies or as a standalone approach. Taleb's original presentation of the AF approach [19] was a loose, qualitative account - as such, there exists no formal presentation of the AF approach to dealing with uncertainty, or the interlinks between the sub-concepts of 'optionality', 'controlling the dispersion of outcomes', 'barbell strategy', 'hormesis', 'redundancy', or 'smallscale experimentation'. We will introduce AF in the next section and then expand on 
the sub-concepts - and our view of their usefulness in dealing with inderterminism after this.

\subsection{The nature of fragility and 'antifragility'}

Taleb [19] establishes the concept of 'antifragility' by juxtaposing it with the more familiar term of 'fragility'. Antifragility is projected as the true antonym of 'fragility', rather than the antonym being 'robustness' as, at first glance, it appears to be. Robustness is, instead, an intermediate state on a continuum between fragility and antifragility (see Fig. 2) and simply implies an ability to withstand harm. Antifragility, by contrast, implies the capacity to gain from harm.

\section{INSERT FIGURE 2 ABOUT HERE}

Fragility can be identified through a concave distribution of outcomes demonstrating exponentially increasing impairment (see Fig. 3) [19 p.272]. Under concavity, positive outcomes are minimal compared to the exponentially increasing level of negative outcomes that could occur. For example, travelling by plane has a concave distribution in terms of its beneficial or negative effects on one's time [19 p.283-284]. A plane will rarely be more than thirty minutes early but has the potential to be days late (or, even worse, not to arrive at all). The potential positive gains are minimal and have a ceiling (the time gains from early arrival on a flight scheduled to take two hours cannot be more than two hours) but the time you could lose is potentially unbounded (especially in the case of the most negative outcome of departure but nonarrival). 
This concave distribution comes about because of the nonlinear effect of harm [19 p.270] and this non-linearity, in turn, provides a further useful way of identifying fragility in contrast to antifragility: fragility exists if the cumulative harm from small shocks is smaller than the harm from a single shock of a size equivalent to these cumulative small shocks [19 p.272]. For example, a porcelain cup can withstand numerous small impacts (for example, being dropped from a small height) but a single impact of a size equivalent to the sum of all the small impacts previously sustained will destroy the cup, which is therefore fragile.

This concept of fragility, incorporated in $\mathrm{AF}$, is of considerable relevance to our theme of dealing with uncertainty in the form of extreme or 'surprise' outcomes or events. The 'fragile' organization is harmed considerably more by a single, extreme, high impact, 'surprise' event than by a succession of small or intermediate-sized events. It is for this reason that IL's deficiency in relation to such events is problematic - because of its focus on an explicit understanding of the underpinning causality of focal events. The occurrence of high-impact events that, a-priori, have no apparent cause is highly problematic if the outcome of the peripheral event is exponentially increasing harm to the organization.

Taleb argues that the way to prepare for such high impact, 'surprise' events is to establish convexity in the organization's experience of their outcomes. With convexity, the distribution of outcomes is benign - the extent of the potential negative outcome is 'clipped' - and possible positive outcomes are exponentially distributed (see Fig. 4). Experience of such a convex distribution of outcomes is, overall, positive. An example from the world of sport is the contracts of top-level football 
coaches. Because football management is an inherently uncertain business it is customary for clauses to be inserted which require the employing club to pay off the full remaining years in the event of an early breaking of the contract that is not mutually agreed. So if he is fired the coach will get a large pay off and will still be free to seek employment elsewhere. Below, we outline methods for developing an organization's exposure to such convex-distributed outcomes.

\section{INSERT FIGURE 4 ABOUT HERE}

\subsection{The value of creating Optionality}

A concept related to convexity - and incorporated into Taleb's AF - is optionality. In the strategy literature, increasing optionality is analogous to building 'flexibility' into the organization and its range of strategic options. 'Flexibility' is also implied by the AF version of the concept but, under AF, optionality is additionally seen as the vector of antifragility [19 p.176] as it is through optionality that convexity is achieved. A simple example is provided by Taleb [19 p.177] in relation to the skewed laws pertaining to tenancy created in some US metropolitan areas in response to issues surrounding the electorates' perceived insecurity of tenancies. This legislation obliges landlords to allow tenants to live in their rented apartments on a more-or-less permanent basis (i.e. until they decide to give up their tenancy) at a predictable rent. It skews the options in favour of the tenant because any large increases in costs (e.g., maintenance) are absorbed by the landlord rather than the tenant and if rents in the city should, generally, fall the tenant has the option of ending the rental agreement to secure a lower rent. The tenant therefore benefits from convexity - his/her potential losses due to an increase in rent are minimal compared to the possible gains in the event that the rental market weakens. Note that it is not necessary to guess what will 
cause an increase in the landlord's costs in order for the tenant to be antifragile in the face of the landlord's increased costs of letting.

In relation to our earlier discussion of causation and determinism, the concept of optionality is important because it is one of the factors rendering AF a truly nondeterministic, non-causal approach to preparation for the future. Optionality renders obsolete intelligence-gathering about the likelihood of future events [19]. It is a shield against our own fallibility in relation to both predicting causation of events and in guessing their outcomes.

\subsection{Controlling the dispersion of outcomes}

As is evident in step two of the BLM process discussed previously, extant planning methods can contain a stress-testing component designed to ascertain the extent to which plans are robust to deviations from what is expected - from the norm or 'average' outcome. The AF approach asserts that such 'sensitivity analysis' is problematic because of the implicit assumption that the magnitude of past events (from which the 'norm' or 'average' is usually estimated) is representative of the magnitude of events that have not yet happened.

Even when 'worst-case scenarios' are used for such stress testing they are usually sampled from the worst that has occurred in the past [19 p.45] and this may not represent the worst that will take place in the future. Attempts to avoid this problem by imagining even worse 'worst-case scenarios', or by imagining different, more extreme events that could potentially happen but have never yet occurred, remain problematic in IL applications. Cognitive psychology has shown that when asked to 
imagine such events people fall back on the heuristics of recency, saliency and familiarity [32] with the result that such stress-testing tends to be limited to evaluating robustness against the last worst-case scenario, or prominent contemporary worries that are featured in the media. As O'Brien [33] argued, in practice, participants in scenario planning workshops using the IL method tend to regularly emphasise economic factors - such as exchange rates, interest rates, and the focal country's economic activity - as uncertainties that are subsequently given prominence in the scenarios that are constructed. Also, recent and current media-emphasised concerns (e.g. of terrorism activities) tend, also, to replicate themselves in to-be-constructed scenarios. O'Brien labelled these practice-recognised issues as 'future myopia'.

By contrast, an AF approach to the future renders stress testing against extreme futures irrelevant - since the focus of the AF approach is to actively position the organization's activities such that the downside outcome of any future event has a protective floor. In addition, organizational positioning within the AF approach also takes advantage of volatility in potential positive outcomes. Thus, dispersal of outcomes in terms of the upside of uncertainty is important but is seen as beneficial rather than problematic.

\subsection{Barbell strategy}

The dispersion of outcomes can be controlled through the adoption of barbell strategies [19 p.161-167], which are one example of convexity. The relation of the barbell strategy to the dispersion of outcomes is seen in that such strategies reject the concept of 'moderate' or 'average' risk as incorporated in 'stress testing' and instead focus on taking no risks whatsoever in areas subject to potentially large-scale negative 
outcomes and, in contrast, taking a lot of small risks in areas subject to potentially large-scale positive outcomes.

A simple example of a barbell strategy related to personal finance is keeping $90 \%$ of funds in non-volatile cash deposits and the remaining $10 \%$ in very risky investments that have a small chance of a large payoff [19 p.161]. This well illustrates the convexity of barbell strategies as the potential losses can only ever amount to $10 \%$ of total funds whereas the potential gains are very large.

\subsection{Hormesis}

Perhaps the most distinctive aspect of AF compared to other approaches to prepare for the future that also emphasise indeterminism [34], or compared to IL and BLM, is its incorporation of the concept of 'hormesis'. Hormesis is defined [19 p.37] in relation to toxicology, as a small dose of a harmful substance that is actually beneficial to the organism [35]. The analogy is inoculation - by subjecting an organism to a small dose of an illness it will become better able to resist that illness in the future.

Hormesis has relatively recently risen to prominence once more through the work of Calbrese and Baldwin [36]. Here, the hormetic process within the human body can be used to inoculate people at risk of exposure to a dangerous substance so as to be more resistant to it should life-threatening exposure occur - for example, rescue workers who clear up after a nuclear accident could be exposed to low levels of radiation in their training in order to build up their resistance levels [35]. Resistance is generated by the fact that an organism exposed to toxins tends to overcompensate its response, thereby building immunity to future exposure. 
The hormesis concept, as incorporated in AF, emphasises that the removal or absence of stressors can be detrimental, even if it appears to be beneficial in the short term. An example is the smoothing of the business cycle that took place during the so-called 'great moderation' of the 1990s and 2000s, lasting until the credit crunch. During this period of pro-longed, continuous expansion (amounting to some 63 quarters of unbroken growth in the UK) any natural contraction that might have occurred ${ }^{1}$ was 'smoothed' away through ultra low interest rates $^{2}$, leading to a large-scale expansion of credit. In the short term, this financial stability appeared beneficial since it appeared that the vagaries of the business cycle had been removed permanently. In actuality, however, in the longer run, this short-term stability gain resulted in a storing up of financial contractions into a single, large and very damaging crunch that the UK had, over time, become increasingly unprepared for.

While this latter example is at the macroeconomic level, the concept of hormesis is of direct relevance to organizations that attempt to remove variation and error - error that may, in fact, contribute to an organization's evolutionary strengthening. The benefits of stressors to an organization's I.T. security systems is one obvious example - since the continual occurrence of external attacks on I.T. systems acts to strengthen the capability to withstand future attacks.

The concept of hormesis, as applied to organizations, can be viewed as evolutionary in nature - increasing the organization's adaptation to the changing environment, making it fitter and better able to survive [35 p.679]. Pech and Oakley [35] have

\footnotetext{
${ }^{1}$ For example, as a result of the bursting of the dotcom bubble around 2000.

${ }^{2}$ At least in terms of what was the norm at that time, if not in terms of what has been seen subsequently.
} 
discussed this evolutionary viewpoint by reference to the work of Greiner [37], suggesting that the evolutionary crises that mark an organization's development can be seen as resulting from a failure to inoculate, through hormesis, at an earlier stage of development. Hormesis is distinct from existing conceptualisations of organizational learning since it implies an additional, strengthened, level of adaptation that could not simply take place through learning alone [35 p.679].

This, organization-level, application of the concept of hormesis is identified by Pech and Oakley [35] as being present in well-known organizations, including Johnson \& Johnson, Kodak and Xerox. Perhaps the most salient lesson that they draw is that managers may 'overprotect' their organizations, thereby risking both longer-term development and adaptation for the sake of short-term tranquillity. Empirical evidence of these effects is still being collected but could come in the form of the survival life-spans of large corporations. For example, it has been shown by de Geus [38] that less than $50 \%$ of the world's largest firms of fifty years ago still exist today. A future important avenue of research could examine the extent to which organizational death results from the removal of stressors.

\subsection{Redundancy}

Within AF, the concept of hormesis is related to that of redundancy. The potential benefits of redundancy are well known and documented - for example in the buffering actions of firms in relation to maintaining stocks of supplies. However, the concept of redundancy, as incorporated in $\mathrm{AF}$, is more than this and places additional emphasis on the possibility of gaining from - not just defending against - uncertainty. In AF, redundancy is seen as a form of investment rather than a defensive strategy [19 p.44]. 
For example, in the event of large-scale, highly impactful 'surprise' events such as a cessation of world supply of a particular material, excess resources held as a buffer by an organization can be sold - thus, in principle, there can be a gain, rather than loss, from 'surprise' events.

One of the most important lessons for preparation for an uncertain future using an $\mathrm{AF}$ approach is to avoid chasing out of the organization the compensations that arise naturally as a result of hormesis. As discussed above, the concept of hormesis implies that over-compensation occurs within the organism (or organization) as a result of exposure to small amounts of harm. These overcompensations, should they be allowed to continue existing, result in a greater ability to resist the same source or class of harm in the future. Thus, this form of redundancy is beneficial - but in the long-run rather than the short-run.

By contrast, in contemporary practice, the increasing sophistication of supply-chain management techniques and the extensive use of technologies such as enterpriseresource planning have acted to remove redundancy in organizational routines. Errorreduction methods such as Six Sigma also act to reduce redundancy. But, as we have seen, from the viewpoint of hormesis, redundancy is a system's naturally-occurring response to the experience of small doses of volatility and is therefore beneficial. In the organizational context, a long period in which no errors occur (because they have been chased out of the system) will degrade any future response to error.

Similarly, under the AF approach to uncertainty, efficiency is seen as synonymous with fragility. The driving-out of inefficiencies in organizations creates tight coupling 
between systems, for example between individuals on a team or stages in a production process. This tight coupling can lead to cascade effects if something, somewhere in the chain, goes wrong. The ripple effect through the system can result in the exponentially increasing harm that is a feature of the concave distribution of outcomes that is associated with fragility. Finkelstein [50 p.164-168] for example shows how a drive for efficiency went too far in the Japanese company Snow Brand Foods, leading to a disastrous outcome. Similarly, in project management, the removal of slack in delivery dates for the individual tasks comprising an overall project can result in almost every aspect of the project lying on the 'critical path' - such that any delay, anywhere, jeopardises the overall completion of the project by the specified date.

A further implication of an AF redundancy analysis is that organizations should avoid excessive amounts of debt, which, from the perspective of AF, is the inverse of redundancy [19 p.44]. For any organization, perhaps especially a relative new, small firm, fluid financial resources give cover over the volatility in performance of the firm. Debt, on the other hand, increases vulnerability to volatility - since a small variation in performance can prevent the firm from servicing its debt and cause it to become bankrupt.

\subsection{Small-scale experimentation (trial and error)}

According to the AF perspective, randomness in the occurrence of events and their outcomes, whether resulting from our epistemological inadequacies or from ontological or 'true randomness', does considerably less damage when it is distributed over time than when it is concentrated [19 p.91]. This analysis implies that organizations should break up investments into small-scale experiments that test the 
market before committing to larger-scale investments. Similarly, organizations can increase antifragility by creating several business units producing different products and ensuring that the markets for their products are only minimally related.

However, there is a trade-off here that has been overlooked within the AF conceptualisation and which may be problematic for some organizations. Running a number of small-scale business units producing different products rather than a single business unit producing one product may result in greater antifragility, but this antifragility may be acquired at the expense of lower specialisation and, therefore, lower productivity and profit in a 'normal' operating environment. The same trade-off is in evidence in the previous discussion of redundancy - efficiency increases productivity and may also increase fragility. However, increases in productivity over the short-term will count for nothing if the organization is then destroyed by lessened antifragility over the long-term.

4.8 Anti-Fragile concepts and alternative approaches to decision making under uncertainty

An important characteristic of AF is that the sub-components that we have discussed interlink with each other, thereby providing the potential of a complete and internallyconsistent approach to dealing with uncertainty. For example, there is a crossover between small-scale experimentation and optionality, with the former effectively facilitating the latter. In discussing the usefulness or otherwise of 'real options reasoning' - a decision-making approach with much similarity to the optionality subcomponent of AF - Barnett and Dunbar [39, p.384] draw on Myers [40] to make the simple but nonetheless crucial point that scaling up investment in particular projects 
incrementally provides decision-making options at each incremental point. An initial small-scale, experimental investment can secure the 'option' later to either continue a particular project or discontinue it upon maturity (upon completion of the first-stage of the project/experimentation). For example, in 1997, Merck signed an agreement with Biogen for Biogen to develop an asthma drug. The agreement involved the scheduled payment of 'stage payments' over several years, during which the actual success of the drug's development by Biogen, the state of the drug market, safety rules, and so on, could all change. The stage payments allowed Merck to retain the option, at each stage, either to 'scale up' or to 'abandon' further involvement in the drug's development. Thus, Merck's upside was unlimited and its downside was capped by the advance payment made to Biogen. Similarly, Cornelius et al. [41] give the example of an oil and gas company that discovered large amounts of natural gas underground in West Africa in the early 1990s, at which time the retail price that could be charged for gas (lower than current prices) meant that its extraction was not viable. However, since that time the natural gas price in both the US and Europe has more than doubled, and the option is now a valuable asset to the company.

In contrast to optionality and real options reasoning, 'effectuation' has been discussed in the entrepreneurship literature. Effectuation is a dynamic way of making decisions - here, the decision-maker is viewed as adaptive to emerging circumstances and also tries to remove risk by alliances with powerful others - i.e., clipping the downside of the possible outcomes of actions taken, keeping options open, etc. Within AF, the search for positions that have a concave distribution of outcomes is in line with this description of the entrepreneur's intuitive way of making sound decisions. The concept of 'effectuation' originates in the work of Sarasvathy [42]. 'Bricolage', as 
discussed in particular by Baker and Nelson [43], is a similar concept which, in fact, features within Taleb's exposition of AF [19 p.23, p.181, p.226, p.348, p.429].

According to Fisher [44, p.1020], successful entrepreneurs tend to focus on what they are willing to lose in making a decision about whether or not to pursue an opportunity. In other words, Fisher views entrepreneurs as adopting a convexity-based approach to decision making under uncertainty, as in AF. Empirical evidence for effectuation and bricolage as underpinning success in entrepreneurial decision making can also be taken as evidence for the potential usefulness of an AF approach, or at least the convexity-based aspect of it. A number of studies have carried out empirical testing of the concept of bricolage and shown it to be a useful concept for describing how firms operate, evidencing varying degrees of beneficial effect on firm performance [45-47]. Sarasvathy [48] has provided empirical evidence related to effectuation through a study of 27 entrepreneurs and cites a number of other empirical studies illustrating its beneficial effect on subsequent business performance.

In summary, there exists no formal presentation of the AF approach to dealing with uncertainty, or the inter-linkages between the sub-concepts of AF. However, the value of each of AF's sub-components of 'optionality', 'controlling the dispersion of outcomes', 'barbell strategy', 'hormesis', 'redundancy', and 'small-scale experimentation' as ways of improving decision making in the face of uncertainty about the future are supported, to a degree, by extant research evidence.

We see AF as either a standalone method or as a complement to standard scenarioplanning approaches. We consider it a better complement for BLM than for IL 
because under IL there is a danger that participants will be seduced by the plausibility of their constructed cause-and-effect scenarios and this may act against the benefits of AF. While this is true of BLM too to a degree, the fully-augmented BLM approach already contains mechanisms by which to weaken the emphasis on cause, such as a consideration of stakeholder motivations. AF takes this dilution of cause, already present in BLM, further by then considering future outcomes without recourse to cause.

When used as a complement to BLM the AF approach allows the organization to place itself in a position to benefit from the unexpected. BLM widens the range of futures considered compared to IL. The AF approach then allows organizations to actively prepare for futures it has not and cannot (because of indeterminism and the fallibility of causal assumptions) consider as part of BLM process. In the next section, we provide practical guidelines to implement the AF approach within a management workshop setting.

\section{Implementation of an AF approach in a workshop setting}

\subsection{Three-step process}

In order to aid preparation for the future within a scenario planning workshop, we advocate a three-step process to analyse and document an organization's fragility to events and outcomes not modelled in a scenario analysis and, further, to consider ways to enhance antifragility:

Step 1) Listing of the organization's current strategies/investments. 
Workshop participants are required at this stage to produce generic names for their organization's strategies and to write them at the top of a piece of paper, followed underneath by a short description summarising the strategy. An example could be Morrisons' (a large UK supermarket) recent decision to enter the online market in which their main rivals such as Tesco and Sainsbury's are already prominent. This strategy might be labelled 'Online retail' and the short description could be 'To enter the market for the online retail and mobile delivery of groceries.' Next, the participants (comprised, in our hypothetical example, of Morrisons' senior management team) would then go on to the second step.

\section{Step 2) Categorising a focal strategy or investment on a continuum between} 'fragile' and 'antifragile'.

This stage involves consideration of the convexity or concavity of the outcomes that could be associated with the strategy or investment under consideration by considering the possible distribution of positive and negative payoffs - regardless of underpinning events or causes.

The focus of the AF approach is on clipping possible downside losses and exposing the organization to potential upside gains. This is achieved by answering the five questions, detailed below, in relation to the focal strategy/investment.

Participants are facilitated in using their answers to these five questions to negotiate and agree on where the considered strategy/investment should be placed on a continuum from 'fragile' to 'antifragile'. 
The questions within Step 2 are:

i) Does the proposed strategy/investment render the organization vulnerable to great harm?

There may be core aspects to an organization's activities that are very sensitive to harm. An example might be Google's core activity of providing free-to-use search engines for internet users. If this aspect of its business were to become somehow devalued - perhaps because the results produced by its search engines had become commercialised to the extent that they were no longer considered unbiased - this would have a very large-scale, deleterious impact on Google and may even jeopardise its continued existence. On the other hand, strategic change in some aspects of Google's business - such as the production of operating systems for smart phones, or its design and production of tablet computers - may produce little exposure to harming the core of Google. Within the latter activities, it may be possible to take greater strategic risks without jeopardising the company's existence.

Answers to this question facilitate a consideration as to which end of the antifragile 'barbell' [19 p.161-167, and discussed earlier] a particular strategy/investment is located. Is it at the end in which no risks should be taken, whatsoever, or is it at the end in which risks can be taken as long as the potential losses can be easily withstood? This consideration primes participants for the following questions:

\section{ii) Is there a cut-off point to potential losses?}

In the example of the 'barbell strategy' for personal finance, discussed earlier, maximum losses could only ever be $10 \%$ of total funds. Consideration as to what may 
cause such a loss is not of relevance in the antifragile approach. In considering answers to the second question of Step 2, participants consider the potential scale of losses associated with a particular strategy or a particular investment.

iii) Are these potential losses of a magnitude that can easily be withstood by the business, without jeopardising its existence? What is the magnitude of potential losses in comparison to the company's cash reserves?

As an insightful example to the purpose of this question, consider the state of the balance sheets of banks in the UK at the point of the recent credit crunch. The total value of loans made by the UK banks at that point-in-time were huge multiples of their cash reserves. As a result of widespread defaults, the UK banks owed more to other banks and organizations than their actual cash reserves. Under normal circumstances businesses in similar situations would fail through bankruptcy. The third question of Step 2 is designed to raise awareness of this possibility.

iv) If the focal investment/strategy were to be expanded, would the potential losses:

a) remain of a similar size?

b) increase in proportion with the increase in potential gains?

c) increase at a rate considerably slower than the increase in potential gains?

d) increase at a rate considerably faster than the increase in potential gains?

The purpose of this fourth question of Step 2 is to try to identify the potential for increasing potential gains in comparison to the potential for increasing potential losses. The focus is on whether potential losses can be clipped at the same time as potential gains are unbounded. 
If convexity is present in a strategy or investment, we might expect an extension of the focal investment or strategy to result in relatively unchanged potential losses but greatly enhanced potential gains. Or, perhaps, potential losses may increase at a rate far slower than potential gains and may still contain a cut-off point. Ideally, potential gains will be unlimited.

v) Are the potential losses associated with the focal investment/strategy de-coupled from the potential losses of other currently-followed strategies, or other investments?

If an organization's activities are correlated (for example, if demand for more than one of the firm's products is from a similar segment of the market) then losses associated with the focal strategy/investment may occur at the same time as losses as a result of other, currently-followed, strategies/investments. It is important, therefore, to consider the scale of losses not only in relation to the considered strategy/investment but also in interaction with other strategies/investments. Whilst a particular strategy or investment may have a clipped downside - and so may not have the potential to bring down the entire organization - the simultaneous failure of multiple strategies/investments, whether clipped or not, could risk the entire organization. The fifth question of Step 2 is aimed at uncovering the extent of potential correlated loss - and addresses the redundancy/buffering aspect of antifragility.

Discussion of answers to these five questions within Step 2 will enable workshop participants to place the focal strategy/investment on the continuum between fragility 
and antifragility. In Step 3, below, participants move on to consider how to create greater antifragility into the focal strategy/investment.

\section{Step 3) Considering how the focal strategy/investment can be made more} antifragile.

In the first part of this step, participants are given a brief presentation on each of the five factors associated with greater antifragility: 'Optionality', 'Barbell', 'Redundancy/buffering', 'Hormesis' and 'Bricolage'. These are the methods by which the dispersion of outcomes can be controlled and losses clipped, as described earlier, while potential gains are unbounded.

In the second part of this step, each participant works alone or in pairs. Participants are asked to develop one example of how the focal strategy/objective can be made more antifragile, by considering each of the five factors in turn. In the final part of this step, ideas are pooled.

One participant's set of examples related to Morrisons' venture into the online grocery retail market, described earlier, might be as follows:

Optionality:

Lease rather than buy delivery vehicles, at least for an initial period. If the service is not successful, cancel the lease.

Barbell:

Offer the service by utilising the online and distribution services of another organization, thus sharing risk and gaining from their expertise - in fact, this is exactly 
what Morrisons did by agreeing an alliance with the online retailer Ocado.

Redundancy/buffering: Have separate warehouses/depots for the developing online business, so as not to hazard the functioning of the supply chain to the existing store-based business currently core to Morrisons' success formula. This might also be considered a Barbell strategy as it seeks to isolate the risks within the new venture so as not to affect the core business.

Hormesis: Use the new online service to switch supplies between neighbouring retail stores or to replenish smaller, local stores which experience high turnover of particular items, in order to simulate and prepare for periods of extremely high demand.

Bricolage: Offer the new online service in one region only, initially. Or, offer online a sub-set of products currently sold in-store (i.e., not the full range available in-store).

\subsection{Optional forth step: stakeholder analysis/ethical considerations}

Because the fully-augmented version of BLM includes an analysis of stakeholder motivations [49], implementing an AF approach as a complement to BLM could allow for a consideration of the ethical aspects of the proposed methods for achieving convexity. This optional fourth step of our proposed AF process considers whether the organization is seeking to achieve greater antifragility by displacing its own fragility onto other organizations or stakeholders. For example, an organization can 
achieve a degree of convexity by placing employees on short-term or 'zero hours' contracts or by using market power to force supplying organizations to bear the costs associated with unpredictable demand for a product or service. These practices may render the focal organization more antifragile but only by rendering employees, or linked organizations, more fragile. By considering the options for achieving antifragility alongside a stakeholder analysis, consideration can be given to the desirability, ethicality and sustainability of the organization's various options for achieving antifragility.

\section{Concluding remarks}

The rapidly increasing complexity of the challenges to which modern organizations are subjected far outpaces the ability to uncover cause, and renders causal guesses provisional, tentative and subject to error. It is therefore, in our view, increasingly important that the methods we use to prepare for the future can cope with the uncertainty brought about by this increasing complexity without relying solely on identifying cause. Most organizations currently employing scenario planning use the basic intuitive logics scenario method but, as we have argued, the backwards logic derivative method carries the benefit of considering the causes of extreme changes in the achievement of an organization's key objectives. This positive aspect, however, will still leave the organization vulnerable to 'surprise' events that can have extreme negative outcomes but that had no, a-priori, obvious causality. Taleb's antifragility conceptualisation - as we have developed and applied it - provides a complementary, or perhaps a complete alternative, approach to dealing with future uncertainty. As such, it enables the decision-maker to actively respond to a very broad range of futures by explicitly embracing indeterminism. Actual events and their outcomes may, 
of course, be subject to determinism in reality but, given our limited capacity to uncover this determinism, we are better off assuming indeterminism and making our preparations on this basis. In short, our guesses as to causes and their outcomes can not be misplaced if we do not make any.

\section{References}

[1] R. Bradfield, G. Wright, G. Burt, G. Cairns, K. Van Der Heijden, The origins and evolution of scenario techniques in long range business planning, Futures 37 (2005) 795-812.

[2] S. K. Evans, Connecting adaptation and strategy: The role of evolutionary theory in scenario planning, Futures 43 (2011) 460-468.

[3] T. J. Chermack, R. A. Swanson, Scenario planning: human resource development's strategic learning tool, Advances in Developing Human Resources 10 (2008) 129-146.

[4] W. Weimer-Jehle, Cross-impact balances: A system-theoretical approach to crossimpact analysis, Technol. Forecast. Soc. Change 73 (2006) 334-361.

[5] P. Bishop, A. Hines, T. Collins, The current state of scenario development: an overview of techniques, Foresight 9 (2007) 5-25.

[6] European Environment Agency, Looking back on looking forward: a review of $\begin{array}{llll}\text { evaluative } & \text { scenario } & \text { literature, available }\end{array}$ http://science.nature.nps.gov/im/monitor/meetings/FtCollins_12/docs/RibeiroT_JMart in 2009 evaluative scenario literature.pdf 2009, viewed 16 December 2012.

[7] T. J. B. M. Postma, F. Liebl, How to improve scenario analysis as a strategic management tool? Technol. Forecast. Soc. Change 72 (2005) 161-173. 
[8] G. Wright, R. Bradfield, G. Cairns, Does the intuitive logics method - and its recent enhancements - produce 'effective' scenarios? Technol. Forecast. Soc. Change 80 (2013) 631-642.

[9] G. Wright P. Goodwin, Decision making and planning under low levels of predictability: Enhancing the scenario method, Int. J. Forecast. 25 (2009) 813-825.

[10] P. Goodwin G. Wright, The limits of forecasting methods in anticipating rare events, Technol. Forecast. Soc. Change 77 (2010) 355-368.

[11] von Plato, J., Creating Modern Probability: Its Mathematics, Physics, and Philosophy in Historical Perspective. Cambridge University Press: Cambridge, UK, 1994.

[12] Hacking, I., The Taming of Chance. Cambridge University Press: Cambridge, UK, 1990.

[13] Laplace, P. S., A Philosophical Essay on Probabilities. Translated by F.W. Truscott and F. L. Emory, Dover Publications, New York, 1951.

[14] Jevons, W. S., The Principles of Science: A treatise on logic and scientific method, Dover Publications, New York, 1958.

[15] McShane, P., Randomness, Statistics, and Emergence, University of Notre Dame Press, Notre Dame, Indiana, 1970.

[16] N. N. Taleb, The Black Swan, Penguin: London, 2008.

[17] G.L.S. Shackle, Epistemics and Economics, Cambridge: Cambridge University Press, 1976.

[18] B. Loasby, Knowledge, Institutions and Evolution in Economics, Routledge: London, 1999. 
[19] N. N. Taleb, AntiFragile, Allen Lane: London, 2012.

[20] P. Schwarz, The art of the long view: Planning for the future in an uncertain world, Currency Doubleday, New York, 1991.

[21] R. Phelps, C. Chan, S. C. Kapsalis, Does scenario planning affect performance? Two explanatory studies, J. of Bus. Res. 51 (2001) 223-232.

[22] R. S. Raubitschek, Multiple scenario analysis and business planning, in: R. Lamb, P. Shrivastava (Eds.), Advances in Strategic Management (Vol. 5), JAI Press, Greenwich, 1988, pp. 181-205.

[23] S. A. van't Klooster, M. B. A. van Asselt, Practising the scenario-axes technique, Futures 38 (2006)15-30.

[24] W. A. Wagenaar, The subjective probability of guilt, in: G. Wright, P.Ayton (Eds.), Subjective Probability, Wiley, Chichester, UK, 118-130.

[25] E. H. Carr, What is history? Pelican: London, 1972.

[26] G. Wright and G. Cairns, Scenario thinking: Practical approaches to the future, Palgrave Macmillan: New York, 2011.

[27] A. Tversky, D. Kahneman, Extensional versus intuitive reasoning: the conjunctive fallacy in probability judgement, Psychol. Rev. 90 (4) (1983) 293-315.

[28] D. Kahneman, Thinking, fast and slow, Allen Lane: London, 2011.

[29] H. I. Ansoff, Managing surprise and discontinuity: strategic response to weak signals, Z. Betr.wirtsch. Forsch 28 (1976) 129-152.

[30] P. J. H. Schoemaker, G. S. Day, S. A. Snyder, Integrating organizational networks, weak signals, strategic radars and scenario planning, Technol. Forecast. Soc. Change 80 (2013) 815-824. 
[31] R. Ramirez, R. Osterman, D. Gronquist, Scenarios and early warnings as dynamic capabilities to frame managerial attention, Technol. Forecast. Soc. Change 80 (2013) 825-838.

[32] R. M. Bradfield, Cognitive barriers in the scenario development process, Advances in Developing Human Resources 10 (2008) 198-215.

[33] F. A. 0'Brien, Scenario planning: lessons for practice from teaching and learning, European Journal of Operational Research 152 (2004) 709-722.

[34] R. Wiltbank, N. Dew, S. Read, S. Sarasvathy, What to do next? The case for nonpredictive strategy, Strategic Management Journal 27 (2006) 981-998.

[35] R. J. Pech, K. E. Oakley, Hormesis: an evolutionary 'predict and prepare' survival mechanism, Leadership and Organization Development Journal 26 (2005) 673-687.

[36] E. J. Calbrese, L. A. Baldwin, Hormesis: a generalizable and unifying hypothesis, Critical Reviews in Toxicology 31 (2001) 353-424.

[37] L. E. Greiner, Evolution and revolution as organizations grow, Harvard Business Review 76 (1998) 55.

[38] A. de Geus, The Living Company, Nicholas Brealey: London, 1999.

[39] M. L. Barnett, R. L. M. Dunbar, Making sense of real options reasoning: An engine of choice that backfires?, in: G. P. Hodgkinson, W. H. Starbuck (Eds.), The Oxford Handbook of Organizational Decision Making, Oxford University Press, Oxford, 2008, pp. 383-398.

[40] S. C. Myers, Determinants of corporate borrowing, Journal of Financial Economics 5 (1977) 147-75. 
[41] P. Cornelius, A. Van De Putte, M. Romani, Three decades of scenario planning in Shell, California Management Review 48 (2005) 92-109.

[42] S. D. Sarasvathy, Causation and Effectuation: Toward a theoretical shift from economic inevitability to entrepreneurial contingency, Academy of Management Review 26 (2001) 243-263.

[43] T. Baker, R. E. Nelson, Creating something from nothing: Resource construction through entrepreneurial bricolage, Administrative Science Quarterly 50 (2005) 329366.

[44] G. Fisher, Effectuation, Causation and Bricolage: A behavioural comparison of emerging theories in entrepreneurship research, Entrepreneurship Theory \& Practice 36 (2012) 1019-1051.

[45] J. Senyard, T. Baker, P. Davidsson, Entrepreneurial bricolage: Towards systematic empirical testing, Frontiers of Entrepreneurship Research 29 (2009) 1-14.

[46] C. U. Ciborra, The platform organization: Recombining strategies, structures and surprises, Organization Science 7 (1996) 103-118.

[47] O. J. Anderson, A bottom-up perspective on innovations, Administration \& Society 40 (2008) 54-78.

[48] S. D. Sarasvathy, Effectuation: Elements of entrepreneurial expertise. New horizons in entrepreneurship research, Edward Elgar Publishing, Cheltenham, UK, 2008.

[49] G. Cairns, M. Sliwa, G.Wright, Problematizing international business futures through a 'critical scenario method', Futures 42 (2010) 971-979.

[50] S. Finkelstein, Why smart executives fail: Four case histories of how people learn the wrong lessons from history, Business History 48 (2006) 153-170. 
[51] F. Phillips, A life in system, Proceedings of the 57th Annual Meeting of the International Society for System Sciences - 2013, HaiPhong, Vietnam. 